\title{
Characteristics of Solvent Fractional Extraction of Beisu Coal
}

\author{
Lin Shi, Guizhen Gong *
}

(School of Chemical Engineering, Xuzhou University of technology, Xuzhou 221018, Jiangsu

\section{Province )}

\begin{abstract}
Fractional extraction of Beisu coal with ethanol and ethyl acetate was carried out to study the existence form and dissolution behavior of the organic matter in Beisu coat. Fourier transform infrared (FTIR) is used for the analysis of various abstract. The results show that the dissolution of organic matter in ethanol and ethyl acetate is a gradual precipitation process. With the increase of the extraction number, the extraction quality of each extract increased first and then decreased, and the extraction rate of these two extracts was the highest in the $3^{\text {rd }}$ time. The quality of the ethanol extract was significantly higher than that of the ethyl acetate extract. These two solvent extraction have good effect on the matter containing $-\mathrm{OH}$, aliphatic side chain structure, aromatic structure. The structure of $-\mathrm{OH}$, benzene ring and $-\mathrm{C}-\mathrm{O}$ is rich in the Beisu coal, but the $\mathrm{C}=\mathrm{O}$ functional groups are less. The study has important basic theoretical significance in the development of high value-added utilization of coal.
\end{abstract}

Keywords: Beishu coal; Ethanol; Ethyl acetate; Fractional extraction; FTIR

\section{北宿煤溶剂分级萃取的特性}

\author{
石林，宫贵贞* \\ (徐州工程学院 化学化工学院, 江苏省 徐州市 221018)
}

摘要: 采用乙醇和乙酸乙酯两种溶剂对北宿煤进行分级萃取, 以研究北宿煤中有机质的存在形式与溶出行为, 并使用红 外光谱分析技术 (FTIR) 各级萃取物进行分析。结果表明: 有机质在乙醇及乙酸乙酯中的溶解, 是一个逐渐析出的过程。 随着萃取次数的增加, 各次萃取物质量先增加后减少, 第 3 次萃取, 两种萃取剂的萃取率均达最高; 乙醇萃取物的质量明显 高于乙酸乙酯萃取物质量; 两种溶剂对含- $0 \mathrm{H}$ 、脂肪侧链、芳香结构和-C-O 结构的物质萃取效果均较好。北宿煤结构中含-OH、 苯环和-C-O 结构较丰富, 而含 $\mathrm{C}=\mathrm{O}$ 官能团较少。该研究在开发煤的高附加值利用方面具有重要的基础理论意义。

关键词: 北宿煤; 乙醇; 乙酸乙酯; 分级萃取; FTIR

中图法分类号： TQ53; 文献标志码 A 引言

煤作为主要的化石资源, 目前其利用包括直接利用即作为燃料、煤的深度加工和经过精细加工生产化 工产品 ${ }^{[1,2]}$ 。以热处理技术为主导的传统煤转化工艺, 无论是燃烧、焦化还是液化或气化, 都有能源使用率 低、污染大且不能获得较高的经济价值。虽然已开发了一些利用煤炭制备二硫化碳、腐植酸、苯多酸等非 燃料利用的方法 ${ }^{[3,4]}$, 但是这些方法对煤种的要求比较高, 不能根本上改变煤炭利用现状, 发挥煤作为化工 产品的作用。随着人们环保意识的加强, 煤炭作为燃料利用的弊端将越来越突出。为了跟随世界发展的趋 势, 应重新确定我国煤炭发展方向, 大力开发煤炭的非燃料利用新途径。将煤处理为清洁燃料及以煤炭为 原料生产具有较高经济价值的产品的最大阻碍是煤的结构复杂。因此, 从分子水平上探索煤的结构 ${ }^{[5]}$, 将 为煤的合理和高效的利用提供理论依据和技术支撑 ${ }^{[6]}$ 。

煤的溶剂萃取是研究煤的组成和结构的常用方法。其是利用溶剂具有的给出-接受电子的能力把煤中的 
一些小分子物质析出。通过分级萃取对不同时间段溶出物进行分析, 不仅可提供研究煤结构的一些信息, 而且可根据萃取物中包含的物质量来判断煤的反应性。煤的溶剂萃取过程一般认为是溶剂首先渗进煤的孔 隙一一分子间作用力被破坏一一整体结构发生变化一一小分子单体溶解在特定的溶剂里。因此, 选择不同 的试剂或者操作来改变这些中间过程, 可以加快萃取速率、提高萃取率及考察萃取机理 ${ }^{[7-10]}$ 。

本研究选用北宿煤为原料, 在温和条件下依次用不同极性的溶剂乙醇和乙酸乙酯进行分级萃取, 并将 萃取物质量的给以比较, 同时原料、萃取物及残渣用 FTIR 进行分析。

\section{1 实验部分}

\section{1 原料、试剂和仪器}

实验选用北宿煤（BSC）作为研究煤样, 经磨碎后过 200 目篎, 置于真空干燥箱中于

$80{ }^{\circ} \mathrm{C}$ 下真空干燥 $24 \mathrm{~h}$, 然后保持真空状态冷却至室温, 取出置于充氮的干燥器内保存备用。其工业 分析和元素分析数据见表 1 。

表 1 煤样的工业分析与元素分析

\begin{tabular}{|c|c|c|c|c|c|c|c|c|}
\hline \multirow{2}{*}{ 煤样 } & \multicolumn{3}{|c|}{ 工业分析 $(W \%)$} & \multicolumn{4}{|c|}{ 元素分析 (W\%,daf) } & \multirow{2}{*}{$\mathrm{S}_{\mathrm{t}, \mathrm{c}}$} \\
\hline & $\mathrm{M}_{\mathrm{ad}}$ & $A_{d}$ & $\mathrm{~V}_{\text {daf }}$ & $\mathrm{C}$ & $\mathrm{H}$ & $\mathrm{N}$ & $\mathrm{O}$ & \\
\hline \multirow{2}{*}{ BSC } & \multirow{2}{*}{6.50} & \multirow{2}{*}{26.19} & \multirow{2}{*}{27.67} & 78.2 & 4.8 & 0.8 & 15.5 & 0.5 \\
\hline & & & & 5 & 2 & 9 & 2 & 2 \\
\hline
\end{tabular}

所用主要仪器设备是上海亚荣生化仪器厂生产的 RE52CS-1 型旋转蒸发仪; 山东鄄城华鲁电热仪器有 限公司生产的 DHT 型摚拌调温电热套; 上海贺德实验设备有限公司生产的 DZF6020 型真空干燥箱; 德国 Bruker 公司生产的 ALPHA 型 FTIR。所用试剂乙醇、乙酸乙酯均为市售分析纯试剂, 经旋转蒸发仪蒸馏后 使用。

\section{2 实验方法}

分别称取 $10 \mathrm{~g}$ 煤样置于两个改良型索氏萃取器中, 分别用乙醇和乙酸乙酯进行分级萃取, 每级萃取 5 $\mathrm{h}$ 。萃取液冷至室温, 用旋转蒸发仪在常压下蒸除大部分溶剂, 然后将浓缩液移至样品瓶中, 使剩余少量 溶剂自然挥发至恒重, 得各级萃取物, 萃余残渣在 $80{ }^{\circ} \mathrm{C}$ 下真空干燥 $24 \mathrm{~h}$ 后称重, 进行下一级萃取。各级 萃取物及最终萃取残渣用 FTIR 分析。

FTIR 分析采用 $\mathrm{KBr}$ 压片, 在 $400^{\sim} 4000 \mathrm{~cm}^{-1}$ 波数范围内扫描。

\section{2 结果与分析}

\section{1 萃取过程分析}

图 1 为乙醇和乙酸乙酯两种萃取溶剂各级萃取物的质量。由图可知, 乙醇每级萃取物的质量均高于乙 酸乙酯。萃取量呈现由低到高, 再由高到低的趋势, 第三次萃取量, 两种溶剂均达最高。经前 5 次萃取后, 第 6 次萃取量最少。其原因可能与煤的溶剂萃取过程有关。煤的萃取过程一般认为是萃取剂对煤的浸润, 渗透, 膨胀的过程。在第三次萃取循环中, 萃取剂完全渗入, 与煤中小分子的接触很完全, 萃取剂的溶解 能力变强, 因而萃取量达到最高。在第三次到第六萃取循环过程中, 煤中的小分子越来越少, 萃取量逐步 
减少, 最终萃取完成。乙酸乙酯萃取物质量小于乙醇萃取物质量的原因, 可能与溶剂的极性及大小有关, 乙醇极性大于乙酸乙酯, 且乙醇的分子小于乙酸乙酯, 乙醇更易于渗透到煤分子内部, 根据相似相溶原理, 煤中的极性化合物更易溶于乙醇。

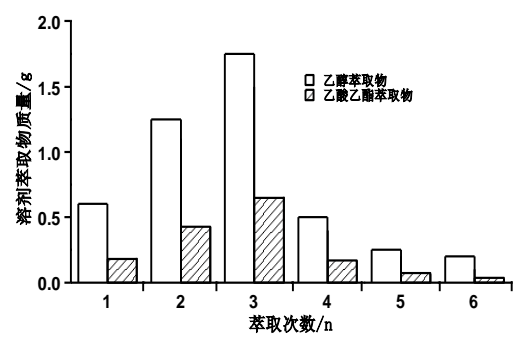

图 1 两种溶剂各级萃取物的质量

\section{2 萃取物的 FTIR 分析}

如图 2 和图 3 所示为各级萃取物的 FTIR 图。图中所示 $3452 \mathrm{~cm}^{-1}$ 左右间宽而强的吸收峰表示-OH 所形 成缔合结构的伸缩振动吸收峰, 在乙醇萃取物及乙酸乙酯萃取物中, 该区域吸收均较强, 表明 $\mathrm{OH}$ 缔合结 构在各级物质中含量均较高。随萃取次数的增加, 该吸收峰强度依次增加。2 $2925 \mathrm{~cm}^{-1}$ 及 $2827 \mathrm{~cm}^{-1}$ 左右的 吸收峰为 $\mathrm{CH}_{3}-$ 和 $-\mathrm{CH}_{2}-$ 的伸缩振动吸收峰。在两种萃取溶剂的各级萃取物中, 第 3 次和第 6 次均较弱, 表明 含 $\mathrm{CH}_{3}$-和 $-\mathrm{CH}_{2}-$ 结构的物质在其中含量较低。 $1637 \mathrm{~cm}^{-1}$ 及 $1561 \mathrm{~cm}^{-1}$ 左右的吸收峰是苯环 $\mathrm{C}=\mathrm{C}$ 骨架伸缩振动, $642 \mathrm{~cm}^{-1}$ 处左右的吸收峰为取代芳烃的吸收, 三处吸收有一共同的变化, 前 5 次有缓慢的递增趋势, 第 4 , 5 次较高, 第 6 次萃取物中又有所降低, 说明含苯环的物质, 随着萃取时间的延长逐渐析出的过程。1130 $\mathrm{cm}^{-1}$ 左右的吸收峰可归结于 $\mathrm{C}-0$ 的伸缩振动吸收峰, 包括醇、醚键和酯类化合物, 两种萃取溶剂的各级萃取物 中, 随着萃取次数的增加逐渐增加。 $\mathrm{C}=0$ 结构在各级萃取物中均不明显, 说明北宿煤中, 含该官能团结构 较少。从各级萃取物的 FTIR 分析可知, 煤中各种物质在乙醇和乙酸乙酯中, 是一个逐渐溶出的过程,

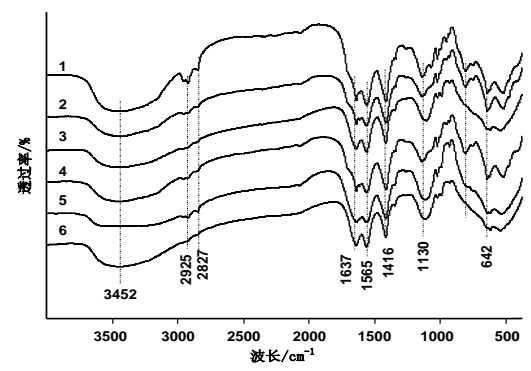

图 2 乙醇各级萃取液的 FTIR 谱图

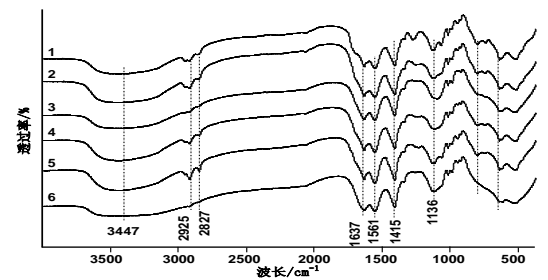

图 3 使用乙酸乙酯萃取所得产物 FTIR 谱图

如图 4 所示为北宿原煤、乙醇萃余煤及乙酸乙酯萃余煤的 FTIR。由图 3 可见，原料和两种萃余残渣的 红外谱图基本一致只是强度有所差异，说明乙醇和乙酸乙酯只是萃取了煤中少部分可萃物，依然有大不部 分物质未被提取出。 


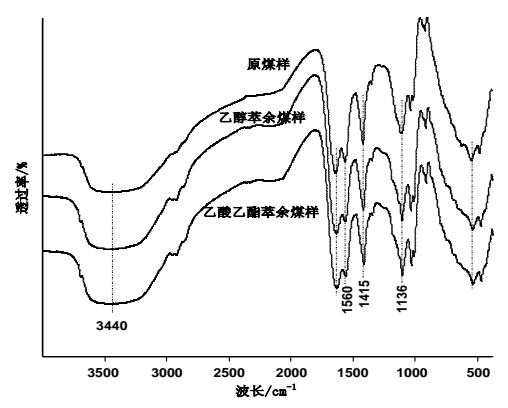

图 4 原煤样与萃余煤的 FTIR 谱图

\section{3 结语}

本研究考察了乙醇和乙酸乙酯对北宿煤的分级萃取效果。随着萃取次数的增加, 各次萃取物质量先增 加, 后减少, 第 3 次萃取, 两种萃取剂的萃取率均达最高, 而且乙醇萃取物的质量明显高于乙酸乙酯萃取 物质量。其原因可能是乙醇为极性分子且分子体积较小可以渗透到煤网络大分子中, 从而使一些有机成分 被提取。FTIR 分析显示, 两种溶剂对含 $-\mathrm{OH}$ 、脂肪侧链、芳香结构和 $-\mathrm{C}-0$ 结构的物质, 萃取效果均较好。 同时也说明, 北宿煤中 $-\mathrm{OH}$ 、脂肪侧链、芳香结构和 $-\mathrm{C}-0$ 结构较丰富, 而 $\mathrm{C}=0$ 结构较少。该研究在开发煤 的高附加值利用方面具有重要的基础理论意义。

\section{4 致谢}

感谢中国建筑材料联合会（2014-M3-4），徐州情报所课题（XKQ016）的资助！

\section{Acknowledgement}

This work was supported by the China Building Material Federation (2014-M3-4) and Xuzhou Information Institute (XKQ016).

\section{参考文献:}

[1] WEIX Y, WaANG X H, ZONG Z M. Extraction of organonitrogen compounds from five Chinese coals with methanol [J]. Energy and Fuels, 2009, 23 (10): 4848-4851.

[2] IU Z W, ZONG Z M, LI J N, et al. Isolation and identification of two bis(2-ethylheptyl) benzenedicarboxylates from Lingwu coal [J]. Energy and Fuels, 2009, 23 (1): 588-590.

[3] GONG G Z, WEI X Y, WANG S L, et al. Preparation of benzene polycarboxylic acids by oxidation of coal with $\mathrm{NaOCl}$ [J]. Advanced Materials Research, 2011, 236-238: 864-867.

[4] SUN L B, WEI X Y, LIU X Q, Zong Z M, Li W. Release of organonitrogen and organosulfur compounds during hydrotreatment of Pocahontas No. 3 coal residue over an activated carbon [J]. Energy \& Fuels, 2009, 23 (10): 5284-52865.

[5] YAO Z S, Wei X Y, Lv J, et al. Oxidation of Shenfu coal with RuO4 and $\mathrm{NaOCl}$ [J]. Energy \& Fuels, 2010, 24 (3): 1801-180.

[6] GONG G Z, Wei X Y, Zong Z M. Separation and analysis of the degradation products of two coals in aqueous NaOCl solution [J]. J. Fuel Chem. Technol., 2012, 40 (1): 1-7.

[7] ZONG Z M, ZhANGJ W, XIE R L, et al. Effect of charring temperature on the composition and solubility of chars formed from rapid heating of Shenfu coal [J]. Energy Sources, Part A: Recovery, Utilization, and Environmental Effects, 2010, 32 (7): 620-627. 
[8] ZhANG L P, HU S, CHEN Q D, et al. Molecular structure characterization of the tetrahydrofuran microwave- extracted portions from three Chinese low-rank coals [J]. Fuel, 2017, (189): 178-185.

[9] SHI D L, WEI X Y, FAN X, et al. Characterizations of the extracts from getting bituminous coal by spectrometries [J]. Energy Fuels, 2013, 27(7): 3709-3717.

[10] CONG X S, ZONG Z M, LI M, et al. Enrichment and identification of cyclized hopanoids from Shengli lignite [J]. Fuel Process Technol, 2015, 134: 399-403. 